\title{
Solvent-Free Powder Synthesis and Thin Film Chemical Vapor Deposition of a Zinc Bipyridyl-Triazolate Framework**
}

\author{
Timothée Stassin, ${ }^{[\mathrm{a}]}$ Ivo Stassen, ${ }^{[a]}$ Nathalie Wauteraerts, ${ }^{[\mathrm{a}]}$ Alexander John Cruz, ${ }^{[\mathrm{a}]}$ \\ Marianne Kräuter, ${ }^{[b]}$ Anna Maria Coclite, ${ }^{[b]}$ Dirk De Vos, ${ }^{[a]}$ and Rob Ameloot ${ }^{*[a]}$
}

\begin{abstract}
MAF-252, a non-porous crystalline coordination polymer, is obtained from the solvent-free reaction of $\mathrm{ZnO}$ with 3(2-pyridyl)-5-(4-pyridyl)-1,2,4-triazole. MAF-252 can be synthesized in powder form and deposited as thin films, starting from
\end{abstract}

\section{Introduction}

Coordination polymers are built from metal ion nodes interconnected by organic linkers. Among these materials, crystalline and microporous metal-organic frameworks (MOFs) have been extensively studied because of their record-breaking specific surface area (up to $7500 \mathrm{~m}^{2} \mathrm{~g}^{-1}$ ) and functionalizable pore interior. ${ }^{[1,2]}$ MOFs are typically synthesized under solvothermal conditions. For example, Yaghi and co-workers prepared zeolitic imidazolate frameworks (ZIFs), a subclass of MOFs, using metal salts and various imidazole linkers in solution. ${ }^{[3]}$ At the same time, Chen and co-workers obtained similar or identical materials, and called these metal-azolate frameworks (MAFs). ${ }^{[4,5]}$ Later, a more sustainable preparation method was demonstrated, based on solvent-free reactions in mixtures of metal oxide and linker (OSFR). ${ }^{[6,7]}$ Interestingly, different polymorphs can be obtained depending on the synthesis conditions, and a number of MOF materials have only been synthesized under solventfree conditions. ${ }^{[8,9]}$

The deposition of new materials as thin films, a prerequisite for their introduction in several applications (e.g., microelectronics), ${ }^{[10]}$ is often achieved through adaptation of powder preparation routes. ${ }^{[11]}$ For example, MOF chemical vapor deposition (MOF-CVD) was inspired by OSFR chemistry. ${ }^{[12]}$ The MOFCVD approach relies on two steps: vapor-phase deposition of an oxide precursor followed by its reaction with the vaporized linker. ${ }^{[9,12-14]}$ Recently, we developed CVD protocols for the

[a] Centre for Membrane Separations, Adsorption, Catalysis and Spectroscopy for Sustainable Solutions (CMACS), KU Leuven,

Celestijnenlaan 200F box 2454, 3000 Leuven, Belgium

E-Mail: rob.ameloot@kuleuven.be http://www.amelootgroup.org

[b] Institute of Solid State Physics, Graz University of Technology, Petersgasse 16, 8010 Graz, Austria

[**] A previous version of this manuscript has been deposited on a preprint server (https://doi.org/10.26434/chemrxiv.9916622.v1).

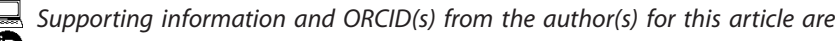
available on the WWW under https://doi.org/10.1002/ejic.201901051.
ZnO powder and layers, respectively. Chemical vapor deposition (CVD) of MAF-252 enables conformal and patterned thin films, even on high aspect ratio features.

microporous zinc 2-methylimidazolate $\left\{\left[\mathrm{Zn}(\mathrm{mlm})_{2}\right]\right\}$ MAF-4 (also known as ZIF-8) and mesoporous zinc 2-ethylimidazolate $\left\{\left[\mathrm{Zn}(\mathrm{elm})_{2}\right]\right\}$ MAF- $6 .{ }^{[12,14]}$ Other candidates to expand the scope of this CVD approach are an isostructural series of MAFs based on 3-(2-pyridyl)-5-(4-pyridyl)-1,2,4-triazole (Hdpt) or the methylated 3-(3-methyl-2-pyridyl)-5-(4-pyridyl)-1,2,4-triazole (Hmdpt). These materials have been reported as MAF-25 $\left\{\left[\mathrm{Co}(\mathrm{dpt})_{2}\right]\right\}, \mathrm{MAF}-26\left\{\left[\mathrm{Co}(\mathrm{mdpt})_{2}\right]\right\}, \mathrm{MAF}-27\left\{\left[\mathrm{Mg}(\mathrm{mdpt})_{2}\right]\right\}$ and MAF-28 $\left\{\left[\mathrm{Zn}(\mathrm{mdpt})_{2}\right]\right\}$. ${ }^{[6,7]}$ Here, we focused on the undocumented reaction of $\mathrm{ZnO}$ with the commercially available linker Hdpt. Surprisingly, the reaction yields a material that is not the Zn-analogue of MAF-25, but rather a novel non-porous crystalline coordination polymer to which we further refer as MAF252 (read: "MAF-25 two"). This study reports the solvent-free formation and characterization of MAF-252 in powder form and as thin films (Figure 1).

a)
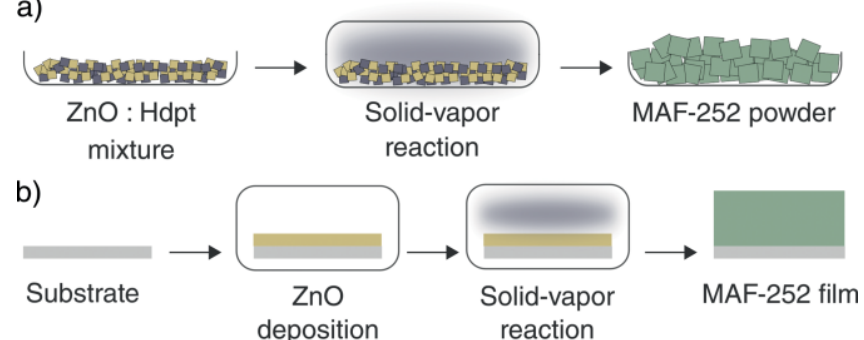

c)

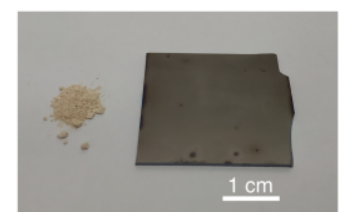

d)

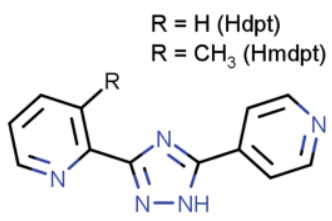

Figure 1. Solvent-free formation of MAF-252 from reaction between $\mathrm{ZnO}$ and 3-(2-pyridyl)-5-(4-pyridyl)-1,2,4-triazole (Hdpt) vapor. (a) Schematic representation of the powder synthesis. (b) Schematic representation of the film deposition. (c) Image of MAF-252 powder and film. (d) Chemical structure of Hdpt and Hmdpt. 


\section{Results and Discussion}

MAF-252 powder was synthesized by heating a mixture of $\mathrm{ZnO}$ and Hdpt at $270{ }^{\circ} \mathrm{C}$ for $16 \mathrm{~h}$ in a glass ampoule, as for the OSFR of other $\mathrm{H}(\mathrm{m}) \mathrm{dpt}$-based MAFs. ${ }^{[6,7]}$ This approach can be translated to a thin film deposition process following a twostep reaction scheme as in MOF-CVD: (1) deposition of a thin (1-15 nm) ZnO layer and (2) reaction of the $\mathrm{ZnO}$ layer with Hdpt vapor. The resulting MAF-252 powder has a beige color, while the MAF-252 films have a homogeneous mirror-like appearance (Figure 1). MAF-252 CVD requires a reaction temperature $\geq 175^{\circ} \mathrm{C}$ (step 2) (Figure S4.1). For this study, MAF-252 thin films were deposited within an hour in a simple glass reactor kept at $200^{\circ} \mathrm{C}$ to ensure a high enough vapor pressure of this low-volatility linker (Figures S3.1 and S3.2). MAF-252 CVD is a very simple and robust film deposition process, while ZIF-8 and MAF-6 CVD require precise control over the atmosphere composition (e.g., relative humidity), as well as the temperature gradient between substrate and reactor to achieve reproducible film morphology. ${ }^{[15]}$

MAF-252 powder and films are crystalline and have similar ATR-FTIR spectra and diffraction patterns (Figures 2a and 2d). No match was found with a known crystal structure (Figure S2.1). The cell parameters and symmetry cannot be unambiguously identified from the powder pattern because of the limited number of reflections and broad peaks. In both cases, no $\mathrm{ZnO}$ is observed by $\mathrm{X}$-ray diffraction, suggesting complete reaction of $\mathrm{ZnO}$ with the linker, as also reported for MAF-28. ${ }^{[6]}$

MAF-252 CVD is sensitive to the ZnO crystallinity and orientation. MAF-252 films grown from amorphous or poorly crystalline $\mathrm{ZnO}$ display a powder-like GIXRD pattern, characteristic of a random crystallite orientation (Figure S4.5). Conversely, diffraction spots instead of continuous rings are observed in the GIXRD pattern of MAF-252 films grown from (100)-oriented and (002)-oriented crystalline $\mathrm{ZnO}$ layers, suggesting some degree of crystallite orientation (Figures $2 \mathrm{e}$ and S4.5). These observa- tions are reflected in the film morphology. The morphology of MAF-252 films grown from non-oriented $\mathrm{ZnO}$ and powders as observed by SEM is identical and consists of elongated micronsized crystallites, as for MAF-27 $\left\{\left[\mathrm{Mg}(\mathrm{mdpt})_{2}\right]\right\}$ (Figure 2c). ${ }^{[6]}$ Films grown from oriented $\mathrm{ZnO}$ display larger crystallites lying parallel to the surface, that we believe result in the observed out-of-plane orientation in the GIXRD patterns. The formation of these large crystallites likely stems from a difference in MAF252 nucleation, growth and crystallite ripening for the different types of ZnO precursor, as observed elsewhere for ZIF-8 CVD. ${ }^{[15]}$ MAF-252 CVD is insensitive to the substrate surface chemistry: films with identical morphology can be deposited on $\mathrm{Si}, \mathrm{Au}$, and $\mathrm{TiO}_{2}$ (Figure S4.7).

Thermogravimetric analysis of MAF-252 in air shows no weight loss below $400^{\circ} \mathrm{C}$ (Figure $2 \mathrm{~b}$ ). Above $400{ }^{\circ} \mathrm{C}$, the material decomposes to $\mathrm{ZnO}$. Since the observed weight loss (83\%) matches the weight loss expected for $\left[\mathrm{Zn}(\mathrm{dpt})_{2}\right](84 \%)$, MAF-252 likely has this chemical formula, similar to MAF-25 $\left\{\left[\mathrm{Co}(\mathrm{dpt})_{2}\right]\right\}$ and MAF-28 $\left\{\left[\mathrm{Zn}(\mathrm{mdpt})_{2}\right]\right\}$ prepared under the same conditions (Table S5.1). ${ }^{[6,7]}$ Temperature-dependent PXRD and SEM confirm the degradation of MAF-252 above $400{ }^{\circ} \mathrm{C}$ (Figures S5.2-S5.4).

While MAF-25 is porous to both $\mathrm{N}_{2}$ and $\mathrm{CO}_{2}$, no porosity was detected in MAF-252 powder by $\mathrm{N}_{2}$ and $\mathrm{CO}_{2}$ physisorption, and in MAF-252 films by $\mathrm{Kr}$ physisorption and $\mathrm{MeOH}$ ellipsometric porosimetry, even after activation for $12 \mathrm{~h}$ at $350{ }^{\circ} \mathrm{C}$ under dynamic vacuum.

MAF-252 CVD was also investigated as a function of starting ZnO thickness and CVD reaction time by SEM, GIXRD, AFM, and ellipsometry. MAF-252 films grown from $1 \mathrm{~nm}$ of $\mathrm{ZnO}$ show scattered crystals on the surface. Thicker $\mathrm{ZnO}$ layers yield MAF-252 films with full surface coverage (Figures S4.2 and S4.3). Conversion of $\mathrm{ZnO}$ to MAF-252 is paired with a significant thickness increase. Starting from 1, 4 and $11 \mathrm{~nm}$ of $\mathrm{ZnO}$ yields MAF252 films with a thickness of 19, 77, and $109 \mathrm{~nm}$ after $16 \mathrm{~h}$, a)

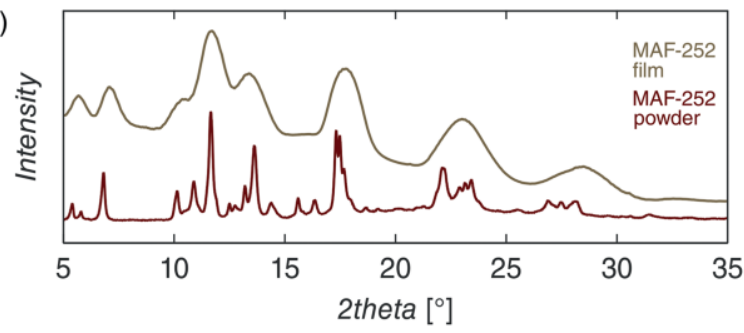

d)

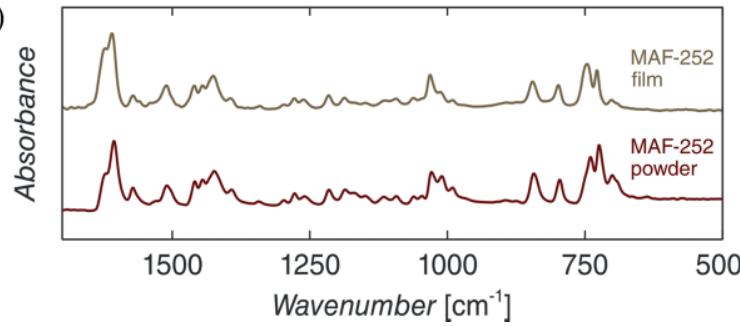

b)

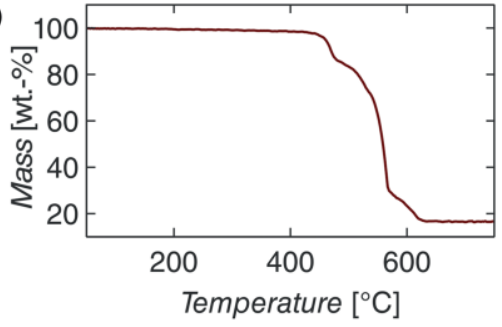

e)

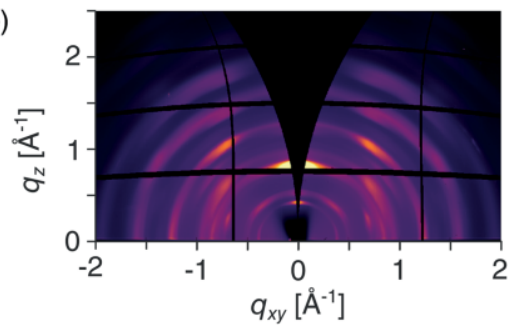

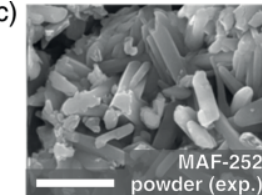
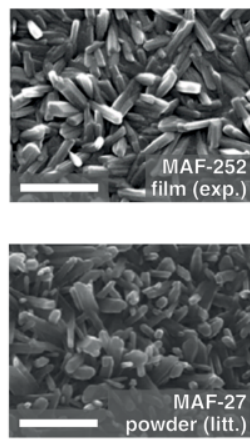

Figure 2. MAF-252 powder and thin film characterization. (a) X-ray diffractogram of MAF-252 powder and film. (b) TGA of MAF-252 powder. (c) SEM images of MAF-252 powder, MAF-252 film, and MAF-27 powder reproduced from ref. ${ }^{[6]}$ with permission from The Royal Society of Chemistry; scale bar $=2 \mu$ m. (d) ATR-FTIR spectra of MAF-252 powder and film. (e) GIXRD plot of an oriented MAF-252 film grown from (002)-oriented ZnO. 
respectively (Figure S4.4). The corresponding film thickness expansion factors are respectively 19, 19 and 10, which is remarkably large for oxide-to-MAF conversions (Table 1). As observed for other materials, linker diffusion hindered by the growing film causes incomplete oxide-to-MAF conversion and a lower apparent expansion factor (10 vs. 19) when starting from thick oxide layers. $^{[12]}$

Table 1. MAF structures and corresponding ZnO-to-MAF film expansion factors.

\begin{tabular}{lllll}
\hline Material & CSD code & Density & \multicolumn{2}{l}{ Film expansion factor ${ }^{[a]}$} \\
& & {$\left[\mathrm{g} \mathrm{cm}^{-3}\right]$} & Bulk ZnO & ALD ZnO \\
\hline ZIF-8 (MAF-4) & VELVOY & 0.95 & 17 & 12 \\
MAF-6 & MECWOH & 0.77 & 22 & 15 \\
MAF-28 & UYAQER & 1.53 & 24 & 17 \\
MAF-252 & This work & - & - & 19 (exp.) \\
\hline
\end{tabular}

[a] Theoretical values based on the bulk $\mathrm{ZnO}$ density $\left(5.6 \mathrm{~g} \mathrm{~cm}^{-3}\right)$ and measured ALD ZnO density $\left(3.9 \mathrm{~g} \mathrm{~cm}^{-3}\right)$, and the know crystal structure of ZIF-8 (MAF-4), MAF-6, and MAF-28. Experimental value from the ratio of the film thickness of $\mathrm{ZnO}$ precursor and MAF-252 films measured by ellipsometry.

Different growth stages can be observed: smooth and thin amorphous films in the first 10 min likely corresponding to the surface reaction between $\mathrm{ZnO}$ and Hdpt vapor, followed by MAF-252 nucleation and crystallization observed as a steep increase in film thickness and roughness. After $1 \mathrm{~h}$ reaction, the growing MAF-252 film hinders the linker diffusion and further oxide conversion. At the same time, larger crystallites are formed by ripening (Figures 3 and S4.4). A similar growth process was observed for ZIF-8 CVD. ${ }^{[15]}$
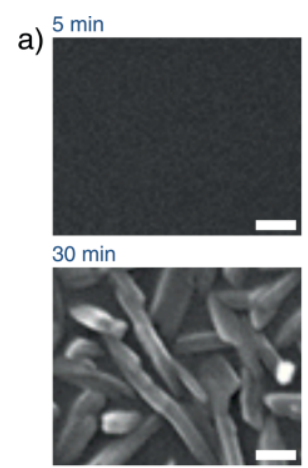

b)

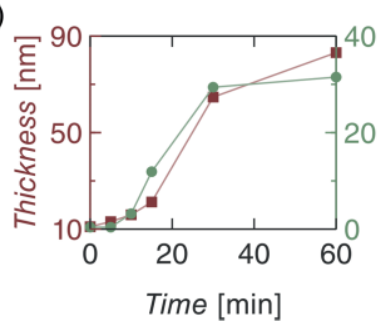

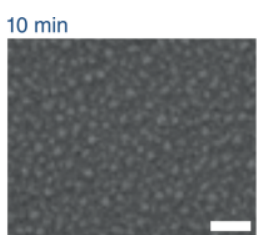

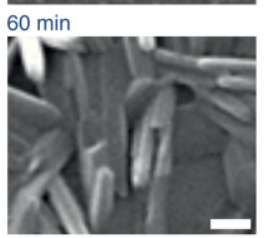

c)

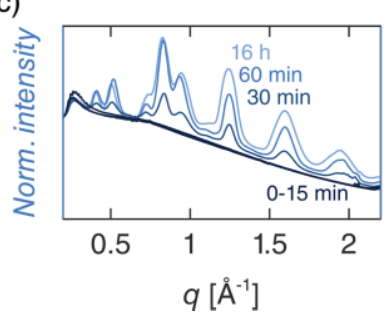

Figure 3. Time evolution of MAF-252 CVD. (a) SEM images, (b) thickness by ellipsometry and RMS roughness by AFM, and (c) diffractograms extracted from GIXRD patterns of MAF-252 films grown from $10 \mathrm{~nm}$ ALD ZnO at various conversion times.

Some applications could benefit from the large film expansion upon conversion of $\mathrm{ZnO}$ to MAF-252 and the high carbon content of the resulting films (e.g., carbonization for use in microsupercapacitors). For integration into microelectronics, com- patibility of the deposition process with typical microfabrication steps is critical. Therefore, the conformality of MAF-252 CVD and film patterning were evaluated (Figure 4). Conformal $\mathrm{ZnO}$ precursor layers were deposited by atomic layer deposition (ALD) on high aspect ratio (25:1) silicon micropillars and converted to conformal MAF-252 films over the whole micropillar length. $\mathrm{ZnO}$ was also patterned on a flat substrate by lithography and converted to MAF-252. The original pattern was maintained with high fidelity.

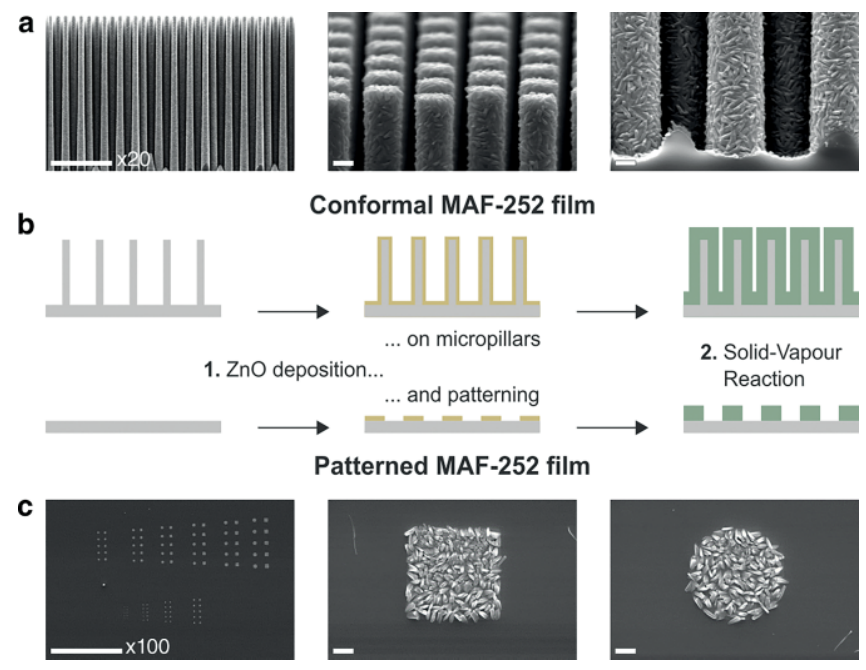

Figure 4. MAF-252 CVD compatibility with typical microfabrication steps. (a) SEM images of MAF-252 films deposited conformally on high aspect ratio Si micropillars. (b) Schematic representation of MAF-252 CVD on high aspect ratio micropillars and film patterning. (c) SEM images of patterned MAF-252 films; scale bar $=1 \mu \mathrm{m}$.

\section{Conclusions}

The formation of the non-porous crystalline coordination polymer MAF-252 from ZnO and a commercially available linker is an example of the potential of solvent-free, vapor-phase processing. MAF-252 CVD is compatible with microfabrication. While the crystal structure of MAF-252 remains to be solved, its integration in applications could benefit from the robustness and the large film expansion factor of the deposition process.

\section{Experimental Section}

Supporting Information (see footnote on the first page of this article): Material and methods can be found in the Supporting Information.

\section{Acknowledgments}

T. S., N. W., and I. S. thank the Research Foundation Flanders (FWO) for a SB-PhD fellowship (1S53316N and 1SB7919N) and for a postdoctoral fellowship (and 12L5417N). T. S. is grateful to Roland Resel and Sabina Rodríguez-Hermida for fruitful scientific discussions. R. A. acknowledges the funding from the European Research Council (No. 716472, acronym: VAPORE) and the Research Foundation Flanders (FWO) for funding in the research 
projects G083016N, G0E6319N, and $1501618 \mathrm{~N}$ and the infrastructure project $\mathrm{GOH} 0716 \mathrm{~N}$. This study is a result of the lead project Porous Materials @ Work (Graz University of Technology, Austria). We acknowledge the Elettra Synchrotron Trieste for allocation of beamtime and thank Luisa Barba and Nicola Demitri for assistance in using beamline XRD1. Min Tu and Helge Reinsch are acknowledged for optimizing the $\mathrm{ZnO}$ patterning protocol and for attempting structure refinement, respectively. High aspect ratio Si micropillars were gracefully provided by imec (Leuven, BE). T. S. thanks the Triumph community for editorial support.

Keywords: Coordination polymers - Chemical vapor deposition - Thin films - Microfabrication · Solvent-free reactions

[1] I. M. Hönicke, I. Senkovska, V. Bon, I. A. Baburin, N. Bönisch, S. Raschke, J. D. Evans, S. Kaskel, Angew. Chem. Int. Ed. 2018, 57, 13780-13783; Angew. Chem. 2018, 130, 13976.

[2] H. Furukawa, K. E. Cordova, M. O'Keeffe, O. M. Yaghi, Science 2013, 341, 1230444.

[3] K. S. Park, Z. Ni, A. P. Cote, J. Y. Choi, R. Huang, F. J. Uribe-Romo, H. K. Chae, M. O'Keeffe, O. M. Yaghi, Proc. Natl. Acad. Sci. USA 2006, 103, 10186-10191.

[4] X.-C. Huang, Y.-Y. Lin, J.-P. Zhang, X.-M. Chen, Angew. Chem. Int. Ed. 2006, 45, 1557-1559; Angew. Chem. 2006, 118, 1587.

[5] A.-X. Zhu, R.-B. Lin, X.-L. Qi, Y. Liu, Y.-Y. Lin, J.-P. Zhang, X.-M. Chen, Microporous Mesoporous Mater. 2012, 157, 42-49.
[6] J.-B. Lin, R.-B. Lin, X.-N. Cheng, J.-P. Zhang, X.-M. Chen, Chem. Commun. 2011, 47, 9185-9187.

[7] J.-B. Lin, J.-P. Zhang, X.-M. Chen, J. Am. Chem. Soc. 2010, 132, 6654-6656.

[8] J. López-Cabrelles, J. Romero, G. Abellán, M. Giménez-Marqués, M. Palomino, S. Valencia, F. Rey, G. Mínguez Espallargas, J. Am. Chem. Soc. 2019, $141,7173-7180$

[9] T. Stassin, S. Rodríguez-Hemida, B. Schrode, A. J. Cruz, F. Carraro, D. Kravchenko, V. Creemers, I. Stassen, T. Hauffman, D. E. D. Vos, et al., Chem. Commun. 2019, 55, 10056-10059.

[10] I. Stassen, N. Burtch, A. Talin, P. Falcaro, M. Allendorf, R. Ameloot, Chem. Soc. Rev. 2017, 46, 3185-3241.

[11] A. Bétard, R. A. Fischer, Chem. Rev. 2012, 112, 1055-1083.

[12] I. Stassen, M. Styles, G. Grenci, H. V. Gorp, W. Vanderlinden, S. D. Feyter, P. Falcaro, D. D. Vos, P. Vereecken, R. Ameloot, Nat. Mater. 2016, 15, 304310.

[13] M. Krishtab, I. Stassen, T. Stassin, A. J. Cruz, O. O. Okudur, S. Armini, C. Wilson, S. D. Gendt, R. Ameloot, Nat. Commun. 2019, 10, 1-9.

[14] T. Stassin, I. Stassen, J. Marreiros, A. J. Cruz, R. Verbeke, M. Tu, H. Reinsch, M. Dickmann, W. Egger, I. Vankelecom, D. De Vos, R. Ameloot, SolventFree Powder Synthesis and MOF-CVD Thin Films of the Mesoporous Metal-Organic Framework MAF-6, 2019; https://doi.org/10.26434/ chemrxiv.9860891.v1.

[15] A. J. Cruz, I. Stassen, M. Krishtab, K. Marcoen, T. Stassin, S. RodríguezHermida, J. Teyssandier, S. Pletincx, R. Verbeke, V. Rubio-Giménez, S. Tatay, C. Martí-Gastaldo, J. Meersschaut, P. Vereecken, S. De Feyter, T. Hauffman, R. Ameloot, Chem. Mater. 2019 31, 9462-9471; https:// doi.org/10.26434/chemrxiv.8984099.v1. 